\title{
The endomotorsonde
}

\author{
G. VANTRAPPEN, J. D'HAENS, S. VERBEKE, AND J. VANDENBROUCKE \\ From the Department of Internal Medicine, University Hospital of \\ St. Raphaël, Louvain, Belgium
}

The insertion of catheters and other instruments into the duodenum and small intestine is a timeconsuming procedure which is unpleasant for the patient. In recent years, however, motility studies have been facilitated by the use of the radiotelemetering capsule (Farrar, Zworykin, and Baum, 1957) but the major disadvantage of this technique is the inability to control the capsule from outside the body, its progress in the gastrointestinal tract depending exclusively on the gastrointestinal propulsive forces.

\section{THE CAPSULE}

In 1958 it was planned to construct a motorized capsule that would avoid this passive progress of the examining instrument and the idea has now been realized in the instrument called the 'endomotorsonde' (Fig. 1). This is a spindle-shaped capsule which comprises a propulsion apparatus and built-in measuring instruments. An electric micromotor sets in motion a pinion system by which the whole capsule is displaced along a dented nylon thread. A miniature pressure transducer of the strain gauge type is built into the capsule (Vantrappen, D'Haens, Vander Stappen, Verbeke, and Vandenbroucke, 1962) which can also be used to introduce a catheter or other miniature instrument into the small intestine. A micro $p \mathrm{H}$ meter is being constructed for use in the capsule. The length of the capsule is $21 \mathrm{~mm}$.; it has a maximal diameter of $7.6 \mathrm{~mm}$.; and its weight is $2.2 \mathrm{~g}$. (Fig. 2).

\section{THE MOTOR SYSTEM}

The electric micromotor measures $8 \times 4 \times 4 \mathrm{~mm}$. The power supply is a 6 volt 100 to $200 \mathrm{~mA}$ direct current source. The speed of the motor varies between 10,000 and 20,000 r.p.m. The incorporated reduction gear reduces this speed by $1 / 450$ to about 60 to 120 r.p.m. The motor power is $0.2 \mathrm{~g}$. per metre per second and thus the motor is able to convey a weight of $50 \mathrm{~g}$. over a distance of $20 \mathrm{~cm}$. in one minute. The pinion system grasps the dented nylon thread of $0.26 \mathrm{~mm}$. diameter along which the whole capsule moves. The teeth of the dented thread have such dimensions and are adjusted in such a way that the pinion grips the dented thread perfectly. The capsule is able to move forward or backward depending upon the direction of the direct current. The entire capsule is attached to a plastic tuble of $1 \mathrm{~mm}$. diameter. Eight different conduction wires are enclosed in this catheter and conduct the impulses from the commanding unit to the motor and send back signals picked up in the gastrointestinal tract to the recording system.

The patient is examined as follows. A latex bag, partially filled with mercury, is attached to the dented thread, and the day before the examination it is introduced through the nasal cavity into the stomach. Initially

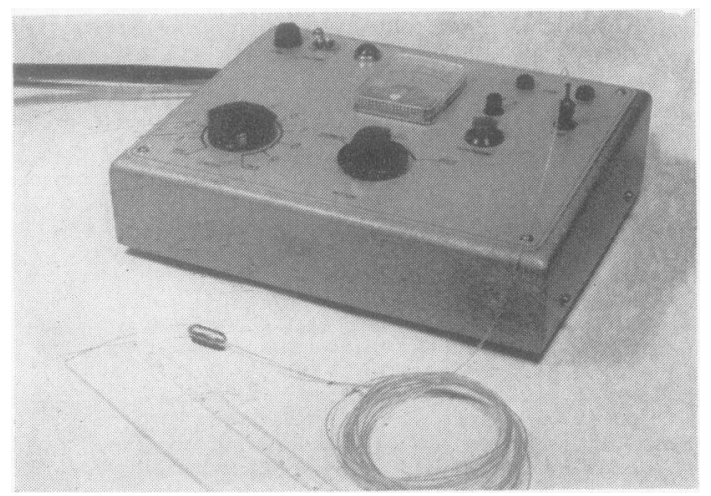

FIG. 1

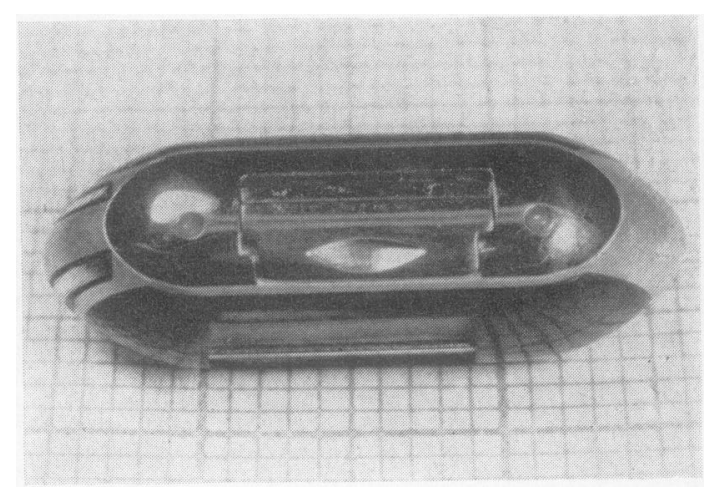

FIG. 2 
the bag was swallowed by the patient but involuntary biting on the thread caused small irregularities sufficient to block the very sensitive traction system. During the first few minutes, the patient's nose may feel uncomfortable due to mucosal irritation. Once the mercury bag has advanced sufficiently into the gastrointestinal tract the actual study is started. Using forceps the thread is caught in the pharynx and drawn through the mouth. Then the dented nylon thread is fitted into the pinion system and the electric motor is started. The upper part of the jejunum is usually reached in about six to 10 minutes. The progress of the capsule can be followed by fluoroscopy. When the study is finished the direction of the electrical current is reversed and the capsule is returned to the mouth. Studies with the endomotorsonde have been performed in more than 40 normal subjects. Neither complications nor accidents have been observed.

\section{THE MEASURING SYSTEM}

The first measuring apparatus is a pressure transducer of the strain gauge type. The pressure changes influence two pairs of strain gauge membranes (Philips PR 9813), measuring $5 / 8.5 \mathrm{~mm}$. Both pairs are built into openings on the lateral surface of the capsule, and are protected by a metallic bar from any direct action of the intestinal wall. The membranes are enclosed in a special plastic holder which fits exactly the shape of the capsule. Both membrane pairs are connected through a Wheatstone bridge and an electrical amplifier (Elema EMT 460) with the recording system.

The physical characteristics of the pressure transducer have been evaluated. The linearity of the pressure registered has been checked by increasing and decreasing the pressure in a step-wise fashion in a closed system. The microtransducer has a linear response up to at least
$100 \mathrm{~cm}$. water. There is no interfering histeresis (Fig. 3).

To determine the frequency response, the microtransducer was placed in a hermetically closed chamber in which pressure changes of constant amplitude were produced with a progressively increasing frequency and were picked up by the microtransducer and registered on the usual recording system. The frequency response of the pressure and registration instruments is such that the amplitude of the pressure changes remains unchanged up to a frequency of three cycles per second (Fig. 4), a response which is adequate for the registration of the physiologically important pressure changes which occur in the duodenum and small intestine.

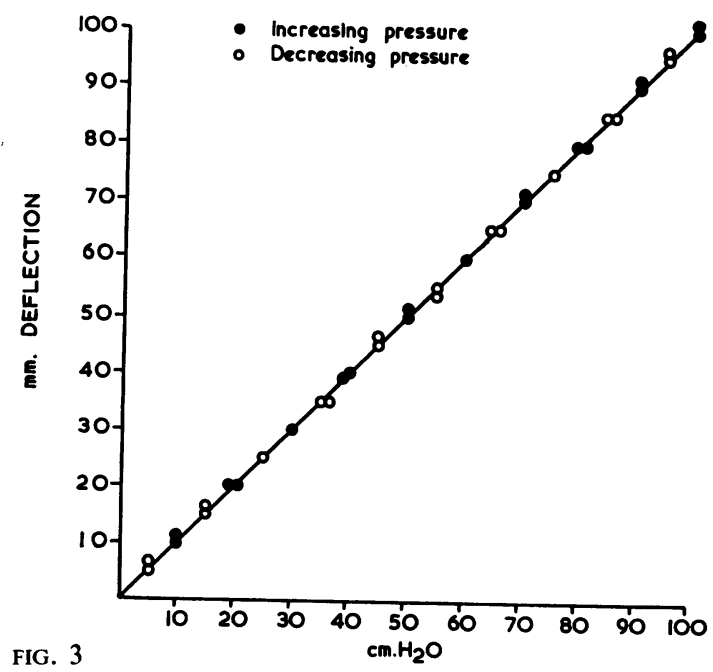

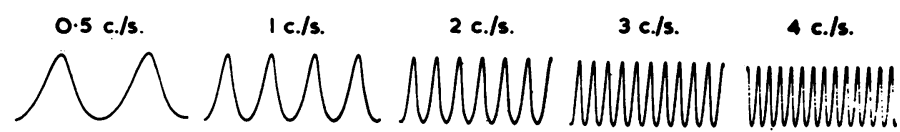
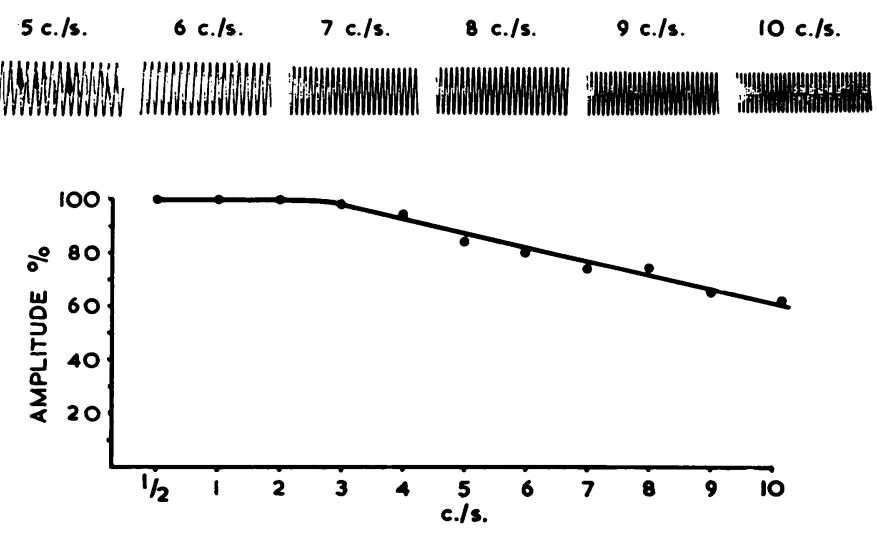

FIG. 4 


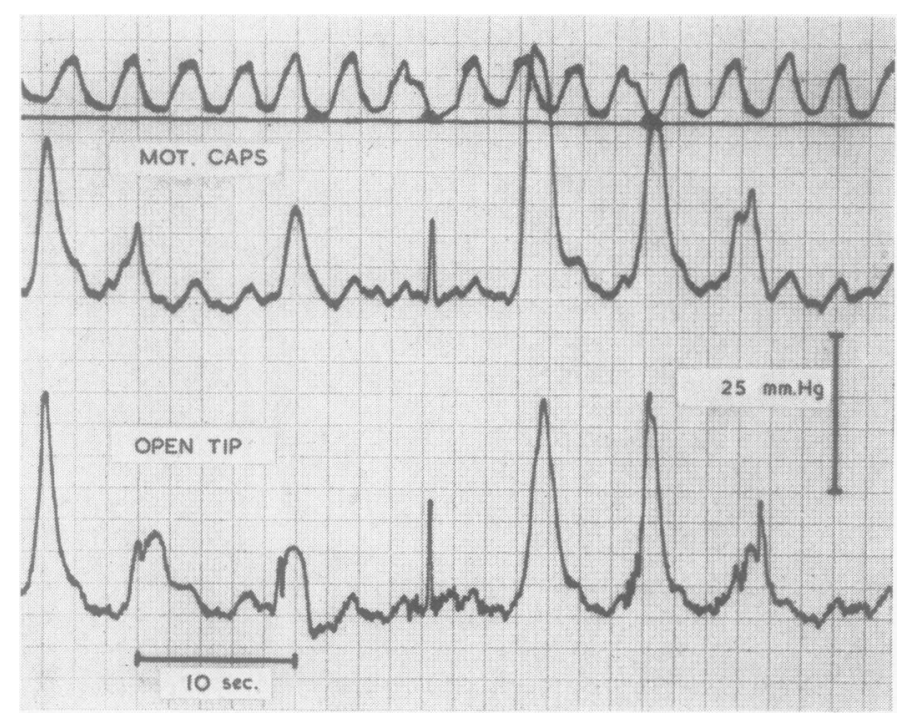

FIG. 5

Simultaneous measurements with the microtransducer and an open-tip catheter system were carried out in the upper jejunum. With both techniques similar pressure tracings were obtained (Fig. 5).

\section{SUMMARY AND CONCLUSIONS}

The endomotorsonde permits miniature measuring instruments to be quickly and easily introduced into the gastrointestinal tract. The capsule is controlled from outside the body. The physical properties of the built-in strain gauges are sufficient to record faithfully the pressure changes in the small bowel.

This technique has no hazards to the patient for no accidents or complications have been encountered. If the micromotor develops any defect the endo- motorsonde can be pulled back through the mouth, and when the connecting thread ruptures it will be eliminated spontaneously.

Metal $p \mathrm{H}$ electrodes are being constructed in the fore end of the capsule, and are being tested in vitro. The endomotorsonde may also be used as a traction system. A catheter can be easily introduced in the small intestine by the micromotor, and aspirations from the content of the small bowel at different segments quickly performed.

\section{REFERENCES}

Farrar, J. T., Zworykin, V. K., and Baum, J. (1957). Science, 126, 975. Vantrappen, G., D'Haens, J., Vander Stappen, G., Verbeke, S., and Vandenbroucke, J. (1962). Presented at the World Congress of Gastroenterology, Munich, May 1962. 